\section{AUTOEFICÁCIA NO ENSINO SUPERIOR E SATISFAÇÃO COM AS EXPERIÊNCIAS ACADÊMICAS: PERCEPÇÕES DE ESTUDANTES DE EDUCAÇÃO FÍSICA}

\author{
SELF-EFFICACY IN HIGHER EDUCATION AND SATISFACTION WITH \\ ACADEMIC EXPERIENCES: PHYSICAL EDUCATION STUDENTS' \\ PERCEPTIONS C
}

\begin{abstract}
AUTOEFICACIA EN LA EDUCACIÓN SUPERIOR Y SATISFACCIÓN CON EXPERIENCIAS ACADÉMICAS: PERCEPCIONES DE ESTUDIANTES DE EDUCACIÓN FÍSICA CP
\end{abstract}

doi' $h$ ttps://doi.org/10.22456/1982-8918.101307

(iD) Vinícius Plentz Oliveira* <viniciusplentz19@gmail.com>

Larissa Fernanda Porto Maciel ${ }^{* *}<$ larimaciel10@gmail.com>

Roberto Tadeu laochite*** <riaochite@gmail.com>

William das Neves Salles ${ }^{* * * *}<$ williamdnsalles@gmail.com>

Juarez Vieira Nascimento* <juarez.nascimento@ufsc.br>

(iD) Alexandra Folle*<afolle_12@hotmail.com>

\footnotetext{
*Universidade Federal de Santa Catarina. Florianópolis, SC, Brasil.

**Universidade do Estado de Santa Catarina. Florianópolis, SC, Brasil.

***Universidade Estadual Paulista Júlio de Mesquita Filho. São Carlos, SP, Brasil.

****Escola Internacional da Universidade Sociedade Educacional de Santa Catarina. Florianópolis, SC, Brasil.
}

\begin{abstract}
Resumo: O objetivo deste estudo descritivo foi analisar a associação entre autoeficácia no Ensino Superior e a satisfação acadêmica de estudantes universitários em Educação Física. Participaram 251 universitários matriculados nos cursos de Licenciatura e Bacharelado em Educação Física de uma universidade pública do estado de Santa Catarina/Brasil. Para a coleta de dados, foram utilizadas a Escala de Autoeficácia no Ensino Superior e a Escala de Satisfação com Experiências Acadêmicas. Os dados foram processados utilizando recursos estatísticos descritivos e inferenciais no software SPSS, versão 25.0. Os resultados mostraram escores médios superiores a sete em todas as dimensões da autoeficácia, com destaque para as dimensões social e gestão. Especificamente, quanto mais o aluno se percebe autoeficaz, mais satisfeito ele fica com a instituição. As evidências ressaltam a importância de pesquisas focadas na relação entre autoeficácia acadêmica e satisfação de estudantes do Ensino Superior para a melhoria da qualidade dos cursos ofertados.
\end{abstract}

Palavras chave: Autoeficácia. Estudantes. Educação Superior. Educação Física.
Recebido em: 25-03-2020 Aprovado em: 14-10-2020 Publicado em: 26-12-2020

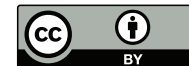

Este é um artigo publicado sob a licença Creative Commons Atribuição 4.0 Internacional (CC BY 4.0).

elSSN: 1982-8918 


\section{INTRODUÇÃO}

O crescente acesso ao Ensino Superior, por si só, não garante o bom desempenho acadêmico ao estudante neste nível educacional. Em função disso, existe uma constante busca das Instituições de Ensino Superior (IES) para compreender os diferentes aspectos que podem estar relacionados à qualidade da formação inicial (TRIGWELL; ASHWIN; MILLAN, 2013), bem como ao sucesso acadêmico e à satisfação dos estudantes com o processo formativo (SANTOS; ZANON; ILHA, 2019).

A autoeficácia $(A E)$, um dos constructos que compõem a Teoria SocialCognitiva, pode ser definida como o julgamento que um indivíduo realiza sobre a própria capacidade de agir em um domínio específico (BANDURA, 1977). Este constructo é importante porque se constitui como base para a motivação, o bemestar e as realizações pessoais futuras (BANDURA, 1977).

No campo da Educação Física, a $A E$ tem sido investigada em domínios diversos, como, por exemplo, da docência (IAOCHITE et al., 2011), na formação de professores (IAOCHITE; SOUZA NETO, 2014; COSTA FILHO; IAOCHITE, 2015; RAMOS et al., 2018; SALLES et al., 2015) e do esporte (IAOCHITE; VIEIRA, 2013). De forma mais específica, no ambiente acadêmico a AE no Ensino Superior referese à capacidade percebida pelo estudante de organizar e executar cursos de ações requeridos para produzir determinadas realizações, considerando as diferentes demandas da formação inicial (POLYDORO; GUERREIRO-CASANOVA, 2010).

No contexto do Ensino Superior, a AE tem sido investigada a partir de cinco dimensões: AE acadêmica (aprender a aplicar os conhecimentos do curso); AE na regulação (criar metas e autorregular as escolhas durante a graduação); AE em ações proativas (ser capaz de aproveitar as oportunidades que a instituição oferece); $A E$ na interação social (relações sociais); e AE na gestão (executar o planejamento e cumprir as demandas do curso) (POLYDORO; GUERREIRO-CASANOVA, 2010).

Estudos sobre a AE no Ensino Superior têm utilizado diferentes instrumentos para coleta das informações, que por sua vez têm revelado diferentes olhares para a influência do constructo nesse contexto. Os resultados encontrados revelam que os estudantes que passam do primeiro ao segundo semestre do curso têm uma redução na percepção de AE (GUERREIRO-CASANOVA; POLYDORO 2011), bem como aqueles que possuem maior percepção de $A E$ tendem a procurar auxílio acadêmico com mais facilidade (XIE; XIE, 2019) e a obter maior sucesso acadêmico (TRIGWELL; ASHWIN; MILLAN, 2013).

Além da $A E$, é importante que se compreenda o nível de satisfação dos estudantes em relação às atividades acadêmicas, de modo a se obter maior entendimento acerca do impacto da formação inicial no desenvolvimento discente e, como consequência, identificar as áreas ou dimensões da formação que necessitem de maior atenção pelos dirigentes institucionais. A satisfação acadêmica, neste sentido, pode ser definida como um estado psicológico resultante da confirmação (ou não) das expectativas do estudante em relação à realidade acadêmica por ele vivenciada (ELLIOTT; SHIN, 2002; JARADEEN et al., 2012). 
Salienta-se que a satisfação acadêmica é influenciada não somente pelas questões objetivas do ambiente, mas também pelas percepções subjetivas de tal meio pelo discente (PEREIRA et al., 2018), as quais são influenciadas pelo nível de AE. A satisfação é considerada um constructo multidimensional que contempla diversas áreas da experiência educacional, tais como a instituição (espaço físico, recursos tecnológicos), o curso (professores, conteúdos, pares) e as oportunidades de desenvolvimento (currículo, iniciativas de formação) (SCHLEICH; POLYDORO; SANTOS, 2006).

A satisfação com as experiências acadêmicas tem sido alvo de estudos de diferentes áreas do conhecimento. De forma geral, os resultados têm apontado que os acadêmicos possuem níveis elevados de satisfação geral com o curso (RAMOS et al., 2015; PEREIRA et al., 2018; SUEHIRO; ANDRADE, 2018), com a instituição e com as oportunidades de desenvolvimento (PEREIRA et al., 2018), revelando, assim, que o sucesso do estudante potencializará seus nível de satisfação, o qual está ligado a toda experiência no contexto educacional (SCHLEICH; POLYDORO; SANTOS, 2006).

Apesar de se identificar a presença de estudos, na literatura consultada, que investigaram a AE no Ensino Superior e a satisfação acadêmica isoladamente, não foram encontradas investigações sobre a relação entre os referidos constructos. Neste sentido, a presente investigação tem como o objetivo analisar a associação entre a AE no Ensino Superior e a satisfação acadêmica de estudantes universitários de Educação Física.

\section{MÉTODO}

\subsection{CARACTERIZAÇÃO DO ESTUDO}

A presente investigação classifica-se como descritiva, com abordagem quantitativa dos dados. A pesquisa descritiva, junto à estratégia associativa, é usada para descrever, sistematicamente, fatos e características presentes em uma determinada população ou área de interesse, ou seja, busca comparações entre grupos e a previsão ou explicação de comportamentos ou processos, enquanto o método quantitativo centra-se na objetividade, considerando que a realidade pode ser compreendida na análise de dados brutos, recolhidos com auxílio de instrumentos padronizados e neutros (ATO; LÓPEZ; BENAVENTE, 2013).

\subsection{PARTICIPANTES}

A população da presente investigação foi composta por 494 estudantes universitários matriculados nos cursos de Licenciatura e Bacharelado em Educação Física de uma universidade pública do estado de Santa Catarina/Brasil. A amostra, cujo processo de seleção foi por conveniência, compreendeu os indivíduos que se encontravam em sala de aula no momento da coleta de dados e aceitaram participar da investigação, totalizando 251 estudantes (143 homens e 108 mulheres) ou 50,8\% da população, com idades entre 18 e 54 anos (23,28 $\pm 5,45$ anos). Verificou-se a predominância $(52,8 \%)$ de estudantes com experiência esportiva anterior $(8,17 \pm 4,96$ 
anos) e sem vínculo empregatício (58,2\%). Dentre os indivíduos que possuíam vínculo empregatício, o tempo médio de experiência foi de 6,16 $\pm 6,39$ anos.

No que diz respeito às características acadêmicas, visualizou-se que a maioria dos estudantes $(53,8 \%)$ era do curso de Licenciatura em Educação Física e se encontrava na primeira metade da formação acadêmica (57,0\%). Além disso, constatou-se que a maior parte dos investigados não tinha participado, até o momento da coleta de dados, de atividades de monitoria acadêmica $(89,6 \%)$, pesquisa $(74,9 \%)$ ou extensão $(70,9 \%)$.

\subsection{INSTRUMENTOS DE COLETA DE DADOS}

Para a coleta dos dados, utilizou-se a Escala de Autoeficácia na Formação Superior (POLYDORO; GUERREIRO-CASANOVA, 2010), que tem como objetivo analisar a percepção de autoeficácia discente por meio de 34 itens, em escala do tipo Likert de 10 pontos, que vai desde um (pouco eficaz) até 10 (muito eficaz). Os itens são organizados em cinco dimensões: (1) acadêmica (ex.: como aprender os conteúdos que são necessários à minha formação?; utilizar estratégias cognitivas para facilitar minha aprendizagem?); (2) social (ex.: expressar minha opinião quando outro colega de sala discorda de mim?; perguntar quando tenho dúvida?); (3) proativas (ex.: contribuir com ideias para a melhoria do meu curso?; atualizar os conhecimentos adquiridos no curso?); (4) regulação (ex.: planejar ações para atingir minhas metas profissionais?; refletir sobre a realização de minhas metas de formação?); e (5) gestão (ex.: esforçar-me nas atividades acadêmicas?; motivarme para fazer as atividades ligadas ao curso?). No processo de validação do instrumento, constatou-se índice geral de consistência interna de 0,94 (variação de 0,80 a 0,81 nas cinco dimensões) (POLYDORO; GUERREIRO-CASANOVA, 2010).

A Escala de Satisfação com as Experiências Acadêmicas (SCHLEICH; POLYDORO; SANTOS, 2006) foi empregada buscando avaliar a satisfação dos acadêmicos com as experiências no âmbito universitário. O instrumento é composto por 35 questões organizadas em três dimensões: (I) satisfação com a instituição (ex.: Recursos e equipamentos audiovisuais disponíveis na instituição; Atendimento e clareza das informações oferecidas pelos funcionários da secretaria); (II) satisfação com o curso (ex.: Relacionamento com os professores; Estratégia de aula utilizada pelos professores); e (III) oportunidade de desenvolvimento (ex.: Diversidade das atividades extracurriculares oferecidas pela instituição; Programa de apoio financeiro oferecido pela instituição). As opções de resposta para cada questão consideram uma escala Likert de cinco pontos, variando entre o nível um (nada satisfeito) e cinco (totalmente satisfeito). Durante o processo de validação, obteve-se um coeficiente alpha de Cronbach de 0,94, indicando uma boa consistência interna de seus itens (SCHLEICH; POLYDORO; SANTOS, 2006).

\subsection{PROCEDIMENTOS DE COLETA DE DADOS}

O projeto de pesquisa foi, inicialmente, apreciado e autorizado pela Chefia do Departamento de Educação Física da universidade investigada e, em seguida, aprovado pelo Comitê de Ética em Pesquisa com Seres Humanos da Universidade 
Estadual de Santa Catarina sobre o parecer 1.357.726/2015. Posteriormente, os professores de ambos os cursos foram contatados para agendamento prévio da coleta de dados durante os horários de aula.

A coleta de dados foi realizada no término do primeiro semestre letivo de 2016, em sala de aula. O pesquisador principal (primeiro autor) explicou aos estudantes os objetivos da pesquisa, deixando claro o caráter voluntário da participação e a possibilidade de desistência dos respondentes. Na sequência, os discentes concordantes leram e assinaram o Termo de Consentimento Livre e Esclarecido (TCLE) e iniciaram a resposta às questões de ambos os instrumentos, com o devido suporte do pesquisador em caso de dúvidas. O tempo de preenchimento dos instrumentos variou entre 20 e 30 minutos.

\subsection{TRATAMENTO DOS DADOS}

O tratamento dos dados foi realizado mediante a aplicação de recursos estatísticos descritivos (média, desvio-padrão e mediana; valores mínimos e máximos; frequências absoluta e relativa, resíduos ajustados) e inferenciais (teste Qui-Quadrado; coeficiente V), com auxílio do software Statistical Package for Social Sciences (SPSS), versão 25.0. As médias de AE e de satisfação discente em cada uma das dimensões foram categorizadas dicotomicamente a partir da observância do percentil 50 (mediana).

O nível de associação entre as percepções de $A E$ e de satisfação dos estudantes investigados foi analisado por meio do teste Qui-quadrado para grupo único com correção de continuidade de Yates (tabelas 2x2). Considerou-se o coeficiente $V$ de Cramer e os resíduos padronizados ajustados (RA) para identificar a força das associações. Adotou-se o nível de significância de $5 \%$ para a interpretação dos resultados da análise inferencial.

\section{RESULTADOS}

\subsection{PERFIL DE AE NO ENSINO SUPERIOR}

A análise das médias de $\mathrm{AE}$ discente dos estudantes investigados indicou predominância de escores médios superiores a sete em todas as dimensões (Figura 1). Observou-se maior concentração dos valores superiores às médias em decorrência da presença de coeficientes negativos de assimetria em todas as dimensões da $A E$, com destaque às dimensões gestão e regulação.

As dimensões social $(8,29 \pm 1,08$ pontos) e gestão $(8,19 \pm 1,43$ pontos $)$ apresentaram os escores médios mais elevados de $A E$, ao passo que a dimensão ações proativas $(7,14 \pm 1,40)$ apresentou os escores médios mais baixos. A distribuição das médias apresentou-se predominantemente assimétrica à esquerda (indicando a presença mais frequente de valores superiores à média) e leptocúrtica (revelando maior incidência de dados em torno da média). Os dados em branco (missing data) foram excluídos da análise do perfil de AE. Não foram observados valores discrepantes (outliers). 
Figura 1 - Perfil de AE no Ensino Superior dos estudantes investigados.

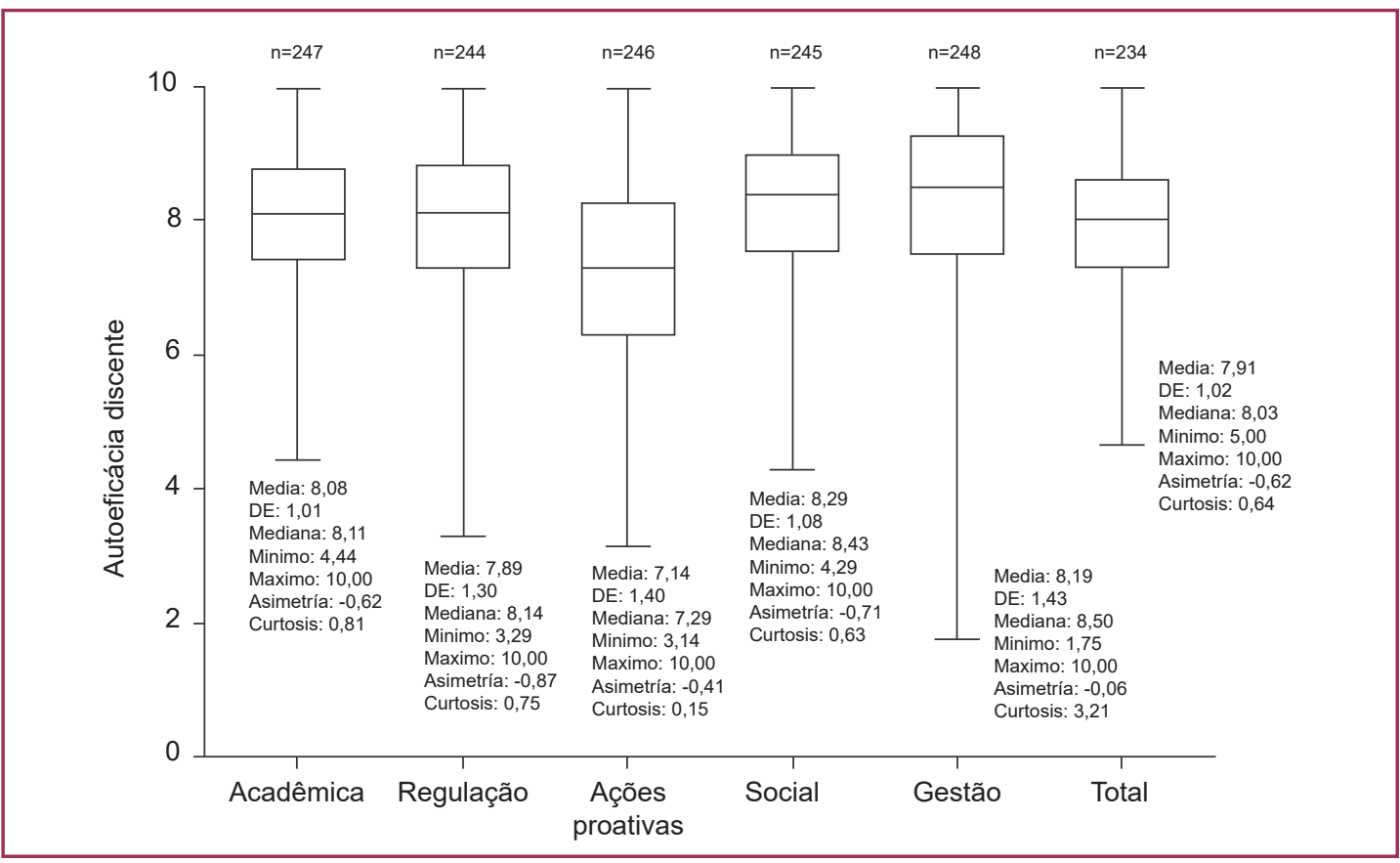

Fonte: Dados da pesquisa

\subsection{PERFIL DE SATISFAÇÃO ACADÊMICA}

A análise das respostas dos estudantes referentes à satisfação acadêmica (Figura 2) revelou médias semelhantes em todas as dimensões, as quais variaram

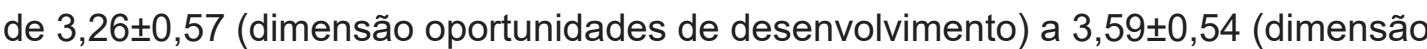
curso). A distribuição das médias apresentou-se praticamente simétrica e mesocúrtica, indicando uma aproximação ao padrão normal. A exemplo do ocorrido na $A E$, os dados em branco foram excluídos da análise do perfil de satisfação. Não foram observados valores discrepantes.

Figura 2 - Perfil de satisfação acadêmica dos estudantes investigados.

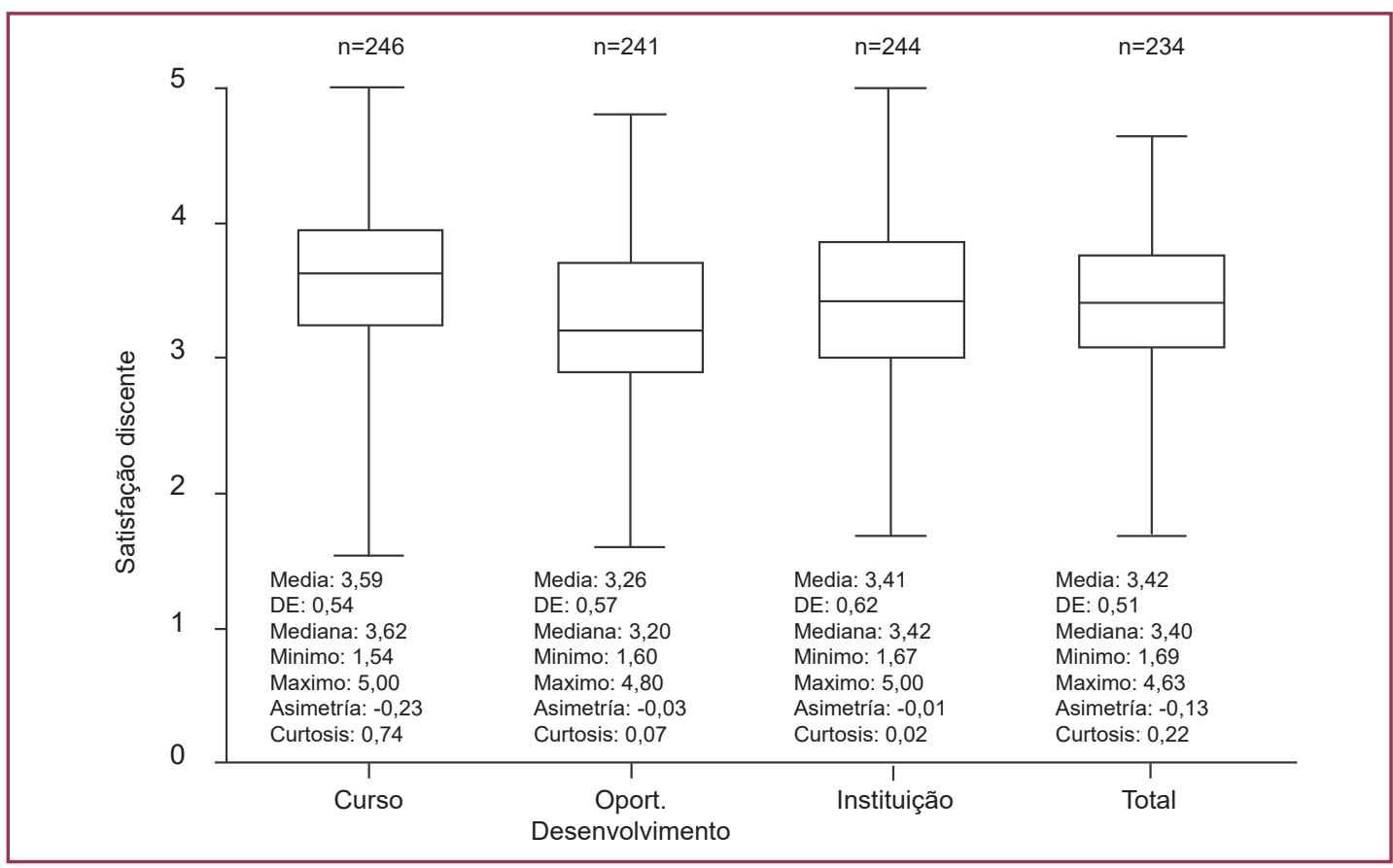

Fonte: Dados da pesquisa 


\subsection{ASSOCIAÇÃO ENTRE AE NO ENSINO SUPERIOR E SATISFAÇÃO ACADÊMICA}

A análise da relação entre as percepções de AE e de satisfação dos estudantes investigados (Tabela 1) revelou predominância de associações estatisticamente significativas $(p \leq \alpha)$ fracas $(0,10 \leq \mathrm{V} \leq 0,20)$ ou moderadas $(0,20 \leq \mathrm{V} \leq 0,40)$ (KOTRLIK; WILLIAMS, 2003) entre as dimensões. As associações mais fortes ocorreram entre a satisfação com o curso e a $A E$ acadêmica $(X 2=20,93 ; V=0,30 ; R A= \pm 4,7)$ e social $(X 2=20,21 ; V=0,30 ; R A= \pm 4,6)$, enquanto as mais fracas foram observadas entre a satisfação com a instituição e a $A E$ social $(X 2=3,83 ; V=0,13 ; R A= \pm 2,1)$ e de gestão $(X 2=4,72 ; V=0,15 ; R A= \pm 2,3)$. $A A E$ proativa foi a dimensão que menos se associou com a satisfação, especialmente por não apresentar relação estatisticamente significativa com as dimensões instituição e geral.

Tabela 1 - Associação entre a AE e a satisfação dos estudantes investigados.

\begin{tabular}{|c|c|c|c|c|}
\hline \multirow[b]{2}{*}{ AE discente } & \multicolumn{4}{|c|}{ Satisfação discente } \\
\hline & Curso & $\begin{array}{l}\text { Oportunidades de } \\
\text { desenvolvimento }\end{array}$ & Instituição & Total \\
\hline \multirow{3}{*}{ Acadêmica } & $X 2=20,93$ & $X 2=7,95$ & $X 2=10,23$ & $X 2=13,00$ \\
\hline & $V=0,30$ & $V=0,19$ & $V=0,22$ & $V=0,25$ \\
\hline & $\mathrm{RA}= \pm 4,7$ & $\mathrm{RA}= \pm 3,0$ & $\mathrm{RA}= \pm 3,3$ & $\mathrm{RA}= \pm 3,7$ \\
\hline \multirow{3}{*}{ Regulação } & $X 2=11,96$ & $X 2=7,64$ & $X 2=8,92$ & $X 2=8,30$ \\
\hline & $V=0,23$ & $V=0,19$ & $V=0,20$ & $V=0,20$ \\
\hline & $\mathrm{RA}= \pm 3,6$ & $\mathrm{RA}= \pm 2,9$ & $R A= \pm 3,1$ & $\mathrm{RA}= \pm 3,0$ \\
\hline \multirow{3}{*}{ Proativa } & $X 2=12,12$ & $X 2=6,55$ & & \\
\hline & $V=0,23$ & $V=0,17$ & $X 2=0,21$ & $X 2=3,09$ \\
\hline & $\mathrm{RA}= \pm 3,6$ & $\mathrm{RA}= \pm 2,7$ & $p=0,65$ & $p=0,08$ \\
\hline \multirow{3}{*}{ Social } & $X 2=20,21$ & $X 2=11,71$ & $X 2=3,83$ & $X 2=10,72$ \\
\hline & $V=0,30$ & $V=0,23$ & $V=0,13$ & $V=0,22$ \\
\hline & $\mathrm{RA}= \pm 4,6$ & $\mathrm{RA}= \pm 3,6$ & $\mathrm{RA}= \pm 2,1$ & $\mathrm{RA}= \pm 3,4$ \\
\hline \multirow{3}{*}{ Gestão } & $X 2=17,03$ & $X 2=11,26$ & $X 2=4,72$ & $X 2=10,63$ \\
\hline & $V=0,27$ & $V=0,23$ & $V=0,15$ & $V=0,22$ \\
\hline & $\mathrm{RA}= \pm 4,3$ & $\mathrm{RA}= \pm 3,5$ & $\mathrm{RA}= \pm 2,3$ & $\mathrm{RA}= \pm 3,4$ \\
\hline \multirow{3}{*}{ Geral } & $X 2=17,21$ & $X 2=11,46$ & $X 2=6,83$ & $X 2=10,74$ \\
\hline & $V=0,28$ & $V=0,23$ & $V=0,18$ & $V=0,23$ \\
\hline & $\mathrm{RA}= \pm 4,3$ & $\mathrm{RA}= \pm 3,5$ & $\mathrm{RA}= \pm 2,7$ & $\mathrm{RA}= \pm 3,4$ \\
\hline
\end{tabular}

Fonte: dados da pesquisa.

\section{DISCUSSÃO}

O objetivo do estudo foi analisar a associação entre a AE no Ensino Superior e a satisfação acadêmica de estudantes universitários de Educação Física. De forma geral, os resultados encontrados revelaram escores médios superiores a 7 em todas as dimensões da $A E$, com destaque para as dimensões social e gestão, o que representa que os participantes têm moderada-elevada AE acadêmica (POLYDORO; GUERREIRO-CASANOVA, 2010).

Esses resultados se aproximam dos obtidos por Santos, Zanon e Ilha (2019), em que a dimensão social da $A E$ foi a mais destacada pelos estudantes. Assim, pode- 
se inferir que estudantes com escores mais elevados para a dimensão social tendem a valorizar mais as relações sociais com a comunidade acadêmica (professores, colegas, funcionários) e a cumprir com as demandas da formação, comprometendose mais com seu futuro profissional e acadêmico (BARDAGI; HUTZ, 2012; SANTOS; ZANON; ILHA, 2019) e desenvolvendo uma visão mais positiva acerca da gestão e demais aspectos da instituição.

Por outro lado, a dimensão AE proativa apresentou a menor média, quando comparada às demais dimensões. Esse resultado é similar ao obtido por estudo com estudantes universitários da primeira e segunda fases (GUERREIRO-CASANOVA; POLYDORO, 2011), o que significa dizer que os estudantes investigados podem, em função de ainda conhecerem pouco as diretrizes e as oportunidades oferecidas pela instituição, aproveitá-las de maneira superficial. Dentre essas oportunidades estão a possibilidade de oferecer sugestões para a melhoria do curso, participar das atividades extracurriculares e conhecer os programas de bolsas e demais recursos disponibilizados para apoiar o corpo discente. Como exemplos destes recursos, destacam-se as bolsas de iniciação científica, de extensão e de suporte financeiro aos alunos de maior vulnerabilidade socioeconômica.

Nesse contexto, pode-se afirmar que um dos exemplos do não aproveitamento das oportunidades que a instituição oferece são as bolsas remuneradas, fato este que pode ser confirmado pela alta porcentagem de estudantes que não possuíam as bolsas de monitoria acadêmica, $89,6 \%$, de pesquisa, $74,9 \%$, e de atividades de extensão, $70,9 \%$, no presente estudo. É relevante destacar que a instituição investigada disponibiliza, anualmente, aproximadamente 100 bolsas remuneradas (ensino, pesquisa e extensão) e que no momento da pesquisa contava com cerca de 500 estudantes regularmente matriculados. É importante ressaltar que mais de $40 \%$ dos investigados possuía vínculo empregatício no momento da coleta de dados, o que também pode ter influenciado no pouco aproveitamento de oportunidades acadêmicas e/ou na falta de ações mais proativas junto à formação inicial.

Por outro lado, a quantidade de bolsas disponível também não possibilita contemplar a todos, algo que pode fazer com que os estudantes já tenham predisposição a não entrar nestes espaços que acabam se tornando restritos. Nesse sentido, faz-se necessário que as instituições busquem otimizar ações que fomentem a participação dos estudantes em atividades complementares não obrigatórias, com o intuito de possibilitar uma formação inicial mais completa (GUERREIRO-CASANOVA; POLYDORO, 2011).

Em relação à satisfação acadêmica, os resultados encontrados evidenciaram altos níveis de satisfação com o curso, na percepção dos estudantes pesquisados. Evidência similar pode ser visualizada em estudos realizados com estudantes de instituições privadas e públicas dos cursos de Psicologia e Enfermagem, respectivamente, nos quais os níveis da dimensão satisfação com o curso apresentaram-se superiores às demais dimensões investigadas (SANTOS; SUEHIRO, 2007; RAMOS et al., 2015). Assim, infere-se que percepções positivas de satisfação estão diretamente relacionadas com a identificação pessoal do estudante com o curso escolhido, visto que estar comprometido com a escolha feita promove 
maior bem-estar psicológico, além de potencializar a avaliação otimista do curso e minimizar as eventuais dificuldades vivenciadas no processo de formação acadêmica (SANTOS; ZANON; ILHA, 2019).

Estudos específicos nesse constructo mostram a relação da satisfação acadêmica com índices de permanência e de diminuição da evasão no Ensino Superior, assim como com aspectos ligados ao engajamento e à realização pessoal e profissional (ALMEIDA, 2019). Além disso, a satisfação acadêmica é um dos determinantes da motivação e do envolvimento estudantil, podendo interferir no desempenho e, consequentemente, na competência do profissional que se inserirá no mercado de trabalho (SOUZA; REINERT, 2010).

No que diz respeito às associações encontradas entre os constructos, visualizou-se associação mais forte das dimensões AE acadêmica e social com a dimensão satisfação com o curso. Tais resultados indicam que a satisfação com o curso pode estar sendo influenciada pela capacidade percebida dos estudantes de participar de atividades extracurriculares, de ajudar e ser ajudado pelos pares e professores e de compreender os procedimentos organizacionais gerais adotados pela instituição para a formação inicial (SCHLEICH; POLYDORO; SANTOS, 2006).

Nesta perspectiva, pode-se refletir que o estudante que possui maiores níveis de AE acadêmica está mais satisfeito com o curso. Nesse caso, estar mais envolvido com a formação inicial do que seus pares menos autoeficazes está relacionado a autopercepções mais elevadas acerca da própria capacidade de aprender, aplicar e demonstrar os conteúdos aprendidos (POLYDORO; GUERREIRO-CASANOVA, 2010).

Da mesma forma, a forte relação entre a satisfação com o curso e a AE social demonstra que a maior capacidade percebida pelo estudante em se relacionar com os pares pode desencadear maior sensação de satisfação com o curso. Nesse sentido, o envolvimento do estudante em círculos de amizades pode aumentar sua percepção de suporte emocional e, ao mesmo tempo, motivar seu comportamento em função das boas ações acadêmicas exibidas pelos amigos mais próximos.

As associações entre a satisfação com a instituição e as dimensões $A E$ social e gestão também se revelaram fortes no presente estudo. Para Mainardes e Domingues (2010), os serviços essenciais (equipe pedagógica, setor administrativo e métodos de ensino) podem influenciar sobremaneira a satisfação dos estudantes com as instituições de Ensino Superior. Neste sentido, considerando os elevados escores apresentados pelos discentes investigados na dimensão social da $\mathrm{AE}$, compreendese a forte relação estabelecida entre esta dimensão e a satisfação com a instituição. A socialização no Ensino Superior tem sido objeto de estudos que, ao discutirem este processo no contexto universitário, estimulam a reflexão à luz de perspectivas teóricas diversas que consideram as instituições universitárias como essenciais para o desenvolvimento humano e profissional dos discentes (STECANELA; MARIN, 2018; SANTOS; ZANON; ILHA, 2019).

Ao mesmo tempo, a elevada capacidade percebida (AE) pelos estudantes em gerenciar as demandas da própria formação possivelmente influenciou positivamente 
sua percepção geral a respeito da IES. A partir do momento em que aqueles estudantes que conseguem gerir bem o próprio tempo têm condições de estabelecer um "retrato" mais amplo e fiel do próprio processo de formação inicial (POLYDORO; GUERREIRO-CASANOVA, 2010), também podem ficar mais conscientes acerca das inúmeras oportunidades de aprendizagem que possuem durante o curso. Isto, provavelmente, influenciou positivamente as percepções deles sobre a instituição investigada.

A dimensão $A E$ proativa, que compreende a capacidade percebida pelo estudante de aproveitar as oportunidades formativas proporcionadas pela instituição (POLYDORO; GUERREIRO-CASANOVA, 2010), apresentou as associações mais fracas com as dimensões da satisfação acadêmica. Nesse caso, verifica-se que os indivíduos que se perceberam com menor AE proativa se mostraram menos satisfeitos com a instituição em relação aos demais, o que pode estar relacionado ao fato de os estudantes não estarem aproveitado suficientemente bem as oportunidades formativas ofertadas.

Em estudo com discentes das áreas de Artes, Ciências e Engenharia, Xie e Xie (2019) encontraram associação entre a percepção de AE e a busca pela ajuda para resoluções de problemas dentro da IES, de modo que os estudantes mais autoeficazes costumam apresentar mais iniciativa para aproveitar os recursos oferecidos pela IES e para procurar auxílio quando necessário. Assim, compreendese que o estudante que dispende esforços para buscar recursos (indivíduos, equipamentos, procedimentos etc.) e solucionar seus problemas se envolve mais com a instituição e, assim, eleva sua satisfação acadêmica ao constatar que sua questão foi resolvida.

\section{CONCLUSÕES}

Em síntese, os resultados mostraram escores médios superiores a 7,00 em todas as dimensões da AE, com destaque para as dimensões social e gestão. Especificamente, quanto mais os discentes se perceberam autoeficazes, mais satisfeitos demonstraram estar com a instituição. No que tange às implicações destes achados para a IES investigada e para as demais IES, ressalta-se que as instituições, ao voltar seu olhar para os estudantes e para sua percepção de AE, necessitam desenvolver ações que permitam maior sentimento de pertencimento do acadêmico em relação às suas escolhas, visando alcançar maiores níveis de satisfação.

O caráter quantitativo deste estudo aponta para algumas limitações. Inicialmente, destaca-se que o processo de amostragem por conveniência adotado nesta investigação implica maior cautela na interpretação dos achados e na comparação com demais estudos relacionados. Dentre elas, o fato de não ter sido explorada uma percepção mais aprofundada e qualitativa dos estudantes. Além disso, este estudo se deteve em analisar a realidade de apenas uma instituição, limitando uma análise mais ampla sobre como a AE pode influenciar a satisfação dos alunos na realidade do ensino superior, da mesma forma como não foram observadas as percepções de outros agentes importantes nesse contexto, tais como professores e gestores. 
Por fim, para avançar em direção a um corpo de literatura mais amplo, sugerese que futuros estudos investiguem mais de uma instituição, bem como utilizem como instrumento de coleta de dados do tipo entrevistas, a observação direta do cotidiano da formação inicial e a triangulação de diferentes fontes de informação (documentos, professores, estudantes, gestores), a fim de se ter uma visão mais integrada da influência do constructo no ambiente de Ensino Superior.

\section{REFERÊNCIAS}

ALMEIDA, Leandro. Ensino Superior: Combinando exigências e apoios. In: ALMEIDA, Leandro S. (ed.). Satisfação acadêmica no ensino superior: desafios e oportunidades. Braga: ADIPSIEDUC, 2019. p. 17-33.

ATO, Manuel, LÓPEZ, Juan J.; BENAVENTE, Ana. Un sistema de clasificación de los diseños de investigación en psicología. Anales de Psicología, v. 29, n. 3, p. 1038-1059, 2013. Disponível em: https://www.redalyc.org/articulo.oa?id=16728244043. Acesso em: 8 de jan. 2020.

BANDURA, Albert. Self-efficacy: toward a unifying theory of behavioral change. Psychological Review, v. 84, n. 2, p. 191, 1977. Disponível em: https://psycnet.apa.org/ record/1977-25733-001. Acesso em: 10 de nov. 2019.

BARDAGI, Marucia Patta; HUTZ, Claudio Simon. Rotina acadêmica e relação com colegas e professores: impacto na evasão universitária. Psico, v. 43, n. 2, p. 174-184, 2012. Disponível em: http://revistaseletronicas.pucrs.br/ojs/index.php/revistapsico/article/ view/7870. Acesso em: 9 de nov. 2019.

COSTA FILHO, Roraima Alves da; IAOCHITE, Roberto Tadeu. Experiências de ensino no estágio supervisionado e autoeficácia para ensinar Educação Física na escola. Journal of Physical Education, v. 26, n. 1, p. 201-11, 2015. Disponível em: http://www.periodicos.uem. br/ojs/index.php/RevEducFis/article/view/24762. Acesso em: 15 maio 2020.

ELLIOT, Kevin; SHIN, Dooyoung. Student satisfaction: An alternative approach to assessing this important concept. Journal of Higher Education Policy and Management, v. 24, n. 1, p. 197-209, 2002. Disponível em: https://www.semanticscholar.org/paper/StudentSatisfaction\%3A-An-alternative-approach-to-Elliott-Shin/00437039a67e03fe8f7f4a2de13c3d b5ddf4abf8. Acesso em: 10 nov. 2019.

GUERREIRO-CASANOVA, Daniela Couto; POLYDORO, Soely Aparecida José. Autoeficácia e integração ao ensino superior: um estudo com estudantes de primeiro ano. Psicologia: Teoria e Prática, v. 13, n. 1, p. 75-88, 2011. Disponível em: http://pepsic.bvsalud.org/scielo. php?script=sci arttext\&pid=S1516-36872011000100006. Acesso em: 10 nov. 2019.

IAOCHITE, Roberto Tadeu; VIEIRA, Roberto Ribeiro. Autoeficácia de técnicos esportivos: um estudo exploratório. Revista Mackenzie de Educação Física e Esporte, v. 12, n. 1, 79-94, 2013. Disponível em: http://editorarevistas.mackenzie.br/index.php/remef/article/ view/4700. Acesso em: 10 nov. 2019.

IAOCHITE, Roberto Tadeu et al. Autoeficácia docente, satisfação e disposição para continuar na docência por professores de Educação Física. Revista Brasileira de Ciências do Esporte, v. 33, n. 4, p. 825-839, 2011. Disponível em: http://www.scielo.br/scielo. php?pid=S0101-32892011000400003\&script=sci abstract\&tlng=pt. Acesso em: 10 nov. 2019. 
IAOCHITE, Roberto Tadeu; SOUZA NETO, Samuel. Strength and sources of self-efficacy beliefs by physical education student teachers. Motriz, v.20, n. 2, p.143-50, 2014. Disponível em: https://www.scielo.br/scielo.php?script=sci_arttext\&pid=S1980-65742014000200143 Acesso em: 17 maio 2020.

JARADEEN, Najah Khaleel et al. Students satisfaction with nursing program.

Bahrain Medical Bulletin, v. 158, n. 690, p. 1-4, 2012. Disponível em: http://www. bahrainmedicalbulletin.com/march 2012/Students Satisfaction.pdf. Acesso em: 12 nov. 2019.

KOTRLIK, Joe W.; WILLIAMS, Heather A. The incorporation of effect size in information technology, learning, and performance research. Information Technology, Learning, and Performance Journal, v. 21, n. 1, p. 1-7, 2003. Disponível em: http://citeseerx.ist.psu.edu/ viewdoc/summary?doi=10.1.1.331.4489. Acesso em: 25 nov. 2019.

MAINARDES, Emerson Wagner; DOMINGUES, Maria José Carvalho de Souza. Satisfação de estudantes em administração de Joinville/SC. Revista Pensamento Contemporâneo em Administração, v. 4, n. 2, p. 76-94, 2010. Disponível em: http://www.spell.org.br/ documentos/ver/30916/satisfacao-de-estudantes-em-administracao-de-jo--- . Acesso em: 5 jan. 2020.

PEREIRA, Aline Fraga et al. Satisfação de estudantes universitários de Educação Física com experiências acadêmicas. Motrivivência, v. 30, n. 53, p. 84-100, 2018. Disponível em: https://periodicos.ufsc.br/index.php/motrivivencia/article/view/2175-8042.2018v30n53p84. Acesso em: 13 dez. 2019.

POLYDORO, Soley Aparecida José; GUERREIRO-CASANOVA, Daniela Couto. Escala de autoeficácia na formação superior: construção e estudo de validação. Avaliação Psicológica, v. 9, n. 2, p. 267-278, 2010. Disponível em: http://pepsic.bvsalud.org/scielo. php?script=sci_arttext\&pid=S1677-04712010000200011. Acesso em: 15 dez. 2019.

RAMOS, Aline Marcelino et al. Satisfação com a experiência acadêmica entre estudantes de graduação em Enfermagem. Texto \& Contexto Enfermagem, v. 24, n. 1, p. 187-195, 2015. Disponível em: http://www.scielo.br/pdf/tce/v24n1/pt 0104-0707-tce-24-01-00187.pdf. Acesso em: 15 dez. 2019.

RAMOS, Valmor et al. Percepção de autoeficácia docente: um estudo sobre as experiências de universitários de Educação Física. Revista Brasileira Ciência e Movimento, v. 26, n. 3, p. 53-62, 2018. Disponível em: https://portalrevistas.ucb.br/index.php/RBCM/article/ view/7758. Acesso em: 3 ago. 2020.

SALLES, William das Neves et al. Autoeficácia discente na formação inicial de estudantes universitários de Educação Física. Movimento, v. 21, n. 4, p. 1083-1097, 2015. Disponível em: https://seer.ufrgs.br/Movimento/article/view/54185. Acesso em: 3 jan. 2020.

SANTOS, Acácia Aparecida Angeli dos; SUEHIRO, Adriana Cristina Boulhoça. Instrumentos de Avaliação da Integração e da Satisfação Acadêmica: Estudo de Validade. Revista Galego-Portuguesa de Psicoloxía e Educacíon, v. 14, n. 1, p. 107-119, 2007. Disponível em: https://core.ac.uk/download/pdf/61900663.pdf. Acesso em: 18 out. 2019.

SANTOS, Acácia Aparecida Angeli dos; ZANON, Cristina; ILHA, Vanessa Domingues. Autoeficácia na formação superior: seu papel preditivo na satisfação com a experiência acadêmica. Estudos de Psicologia, v. 36, n. 1, p. e160077, 2019. Disponível em: http:// www.scielo.br/scielo.php?script=sci abstract\&pid=S0103-166X2019000100201\&lng=en\&nr $\underline{m=i s o \& t l n g=p t . ~ A c e s s o ~ e m: ~} 10$ mar. 2020. 
SCHLEICH, Ana Lúcia Righi; POLYDORO, Soely Aparecida Jorge; SANTOS, Acácia Aparecida Angeli dos. Escala de satisfação com a experiência acadêmica de estudantes do ensino superior. Avaliação psicológica, v. 5, n. 1, p. 11-20, 2006. Disponível em: http:// pepsic.bvsalud.org/scielo.php?script=sci_arttext\&pid=S1677-04712006000100003. Acesso em: 21 nov. 2019.

SOUZA, Saulo Aparecido de; REINERT, José Nilson. Avaliação de um curso de ensino superior através da satisfação/insatisfação discente. Avaliação: Revista da Avaliação da Educação Superior, v.15, n.1, p.159-176, 2010. Disponível em: https://www.scielo.br/pdf/ aval/v15n1/v15n1a09.pdf. Acesso em: 26 jul. 2020.

STECANELA, Nilda; MARIN, Lucas Josias. Convivência acadêmica e formação humana: dimensões de socialização no Ensino Superior. Educação, v. 41, n. 1, p. 93-103, 2018. Disponível em: https://revistaseletronicas.pucrs.br/ojs/index.php/faced/article/view/29552. Acesso em: 26 maio 2020.

SUEHIRO, Adriana Cristina Boulhoça; ANDRADE, Karla Silva. Satisfação com a experiência acadêmica: um estudo com universitários do primeiro ano. Revista Psicologia em Pesquisa, v. 12, n. 2, p. 1-10, 2018. Disponível em: http://pepsic.bvsalud.org/scielo. php?script=sci arttext\&pid=S1982-12472018000200009. Acesso em: 5 jan. 2020.

TRIGWELL, Keith; ASHWIN, Paul; MILLAN, Elena. Evoked prior learning experience and approach to learning as predictors of academic achievement. British Journal of Educational Psychology, v. 83, n. 1, p. 363-378, 2013. Disponível em: https://www.ncbi. nlm.nih.gov/pubmed/23822526. Acesso em: 5 jan. 2020.

XIE, Dengfeng; XIE, Zhangming. Effects of Undergraduates' Academic Self-Efficacy on Their Academic Help-Seeking Behaviors: The Mediating Effect of Professional Commitment and the Moderating Effect of Gender. Journal of College Student Development, v. 60, n. 3, p. 365-371, 2019. Disponível em: https://eric.ed.gov/?id=EJ1219104. Acesso em: 10 fev. 2020. 
Abstract: This descriptive study analyzes the association between self-efficacy in Higher Education and Physical Education students' academic satisfaction. Participants were 251 university students enrolled in the Teacher's and Bachelor's Degrees and Physical Education at a Public University in the state of Santa Catarina, Brazil. The Higher Education Self-Efficacy Scale and the Academic Experience Satisfaction Scale were used for data collection. The data were processed using descriptive and inferential statistical resources of the SPSS software, version 25.0. The results showed mean scores above seven in all dimensions of self-efficacy, with emphasis on the social and management dimensions. Specifically, the more students perceive themselves to be effective, the more satisfied they are with the institution. The evidence underscores the importance of research focused on the relationship between academic self-efficacy and higher education students' satisfaction to improve the quality of the courses offered.

Keywords: Self Efficacy. Students. Higher Education. Physical Education.

Resumen: El objetivo de este estudio descriptivo fue analizar la asociación entre autoeficacia en la Educación Superior y la satisfacción académica de los estudiantes universitarios en Educación Física. Participaron 251 estudiantes universitarios matriculados en los cursos de Licenciatura en Educación Física en una Universidad Pública del estado de Santa Catarina/Brasil. Para la recolección de datos, se utilizaron la Escala de Autoeficacia en la Educación Superior y la Escala de Satisfacción con Experiencias Académicas. Los datos se procesaron utilizando recursos estadísticos descriptivos e inferenciales en el software SPSS, versión 25.0. Los resultados mostraron puntuaciones medias superiores a siete en todas las dimensiones de autoeficacia, con énfasis en las dimensiones social y de gestión. Específicamente, mientras más se percibe el estudiante como autoeficaz, más satisfecho está con la institución. Las evidencias subrayan la importancia de investigaciones centradas en la relación entre autoeficacia académica y satisfacción de los estudiantes de la Educación Superior para mejorar la calidad de los cursos ofrecidos.

Palabras clave: Autoeficacia. Estudiantes. Educación Superior. Educación Física. 


\section{LICENÇA DE USO}

Este é um artigo publicado em acesso aberto (Open Access) sob a licença Creative Commons Atribuição 4.0 Internacional (CC BY 4.0), que permite uso, distribuição e reprodução em qualquer meio, desde que o trabalho original seja corretamente citado. Mais informações em: http://creativecommons.org/licenses/by/4.0

\section{CONFLITO DE INTERESSES}

Os autores declararam que não há conflito de interesses neste trabalho.

\section{CONTRIBUIÇÕES AUTORAIS}

Vinícius Plentz Oliveira: Contribuir substancialmente na interpretação e na discussão dos dados; participar da aprovação da versão final do manuscrito; contribuir na formatação do artigo; contribuir significativamente na escrita do manuscrito; contribuir significativamente na elaboração do conteúdo do manuscrito; elaboração do projeto de pesquisa;

Larissa Fernanda Porto Maciel: Contribuir substancialmente na interpretação e na discussão dos dados; contribuir significativamente na escrita do manuscrito; contribuir significativamente na elaboração do conteúdo do manuscrito; Auxiliar na escrita do referencial teórico. Revisão do Projeto de Pesquisa.

Roberto Tadeu laochite: Participar da aprovação da versão final do manuscrito; contribuir significativamente na escrita do manuscrito; contribuir na formatação do artigo;

William das Neves Salles: Contribuir substancialmente para a concepção e planejamento do projeto de pesquisa; contribuir significativamente na escrita do manuscrito; contribuir substancialmente na interpretação e na discussão dos dados; Elaboração das figuras e tabelas com os resultados da pesquisa.

Juarez Vieira Nascimento: Participar da aprovação da versão final do manuscrito; contribuir significativamente na escrita do manuscrito; contribuir significativamente na elaboração do conteúdo do manuscrito;

Alexandra Folle: Contribuir substancialmente para a concepção e planejamento do projeto de pesquisa; participar da aprovação da versão final do manuscrito. contribuir significativamente na escrita do manuscrito.

\section{FINANCIAMENTO}

O presente trabalho foi realizado sem qualquer apoio financeiro.

\section{ÉTICA EM PESQUISA}

Aprovado pelo Comitê de Ética em Pesquisa com Seres Humanos da Universidade Estadual de Santa Catarina sobre o parecer 1.357.726/2015.

\section{COMO REFERENCIAR}

OLIVEIRA, Vinícius Plentz; MACIEL, Larissa Fernanda Porto; IAOCHITE, Roberto Tadeu, SALLES, William das Neves; NASCIMENTO, Juarez Vieira; FOLLE, Alexandra. Autoeficácia no ensino superior e satisfação com as experiências acadêmicas: percepções de estudantes de educação física. Movimento (Porto 
Alegre), v.26, p. e26087, jan./dez. 2020. Disponível em: https://seer.ufrgs.br/ Movimento/article/view/101307. Acesso em: [dia] [mês abreviado]. [ano]. DOI:

https://doi.org/10.22456/1982-8918.101307

\section{RESPONSABILIBADE EDITORIAL}

Alex Branco Fraga*, Elisandro Schultz Wittizorecki*, Ivone Job*, Mauro Myskiw*, Raquel da Silveira*

*Universidade Federal do Rio Grande do Sul, Escola de Educação Física, Fisioterapia e Dança, Porto Alegre, RS, Brasil 